\title{
Metodologia baseada em recursos computacionais para a introdução de limites no Ensino Médio
}

\author{
Helena Corrêa Ribeiro
}

\author{
André Fabiano Steklain Lisbôa
}

\section{Resumo}

O presente artigo tem como objetivo apresentar uma metodologia de ensino baseada em Recursos Computacionais para a introdução do conceito de limite no ensino médio, utilizando os softwares Geogebra e Maxima. Este trabalho faz parte de uma proposta mais ampla de resgatar o ensino do Cálculo no âmbito escolar, utilizando a tecnologia como uma ferramenta facilitadora no processo ensino-aprendizagem. As ferramentas utilizadas servem para visualização dos conceitos e cálculo algébrico, duas situações que impõem obstáculos ao aprendizado de cálculo pelos estudantes.

Palavras-chave: Cálculo; recursos computacionais; ensino médio.

\section{Abstract}

The goal of this paper is to present a teaching methodology based on computational resources in order to introduce the concept of limit in high school using the softwares Geogebra and Maxima. This work is part of a broader project to rescue the teaching of Calculus in high school using technology as a facilitating tool in the teaching-learning process. These tools are mainly used for the visualization of concepts and algebraic calculations, two situations that impose obstacles to the learning of calculus at this level.

Keywords: Calculus; computacional resources; high school.

\section{Introdução}

O Cálculo é uma ferramenta básica para todos os profissionais que atuam na área de Ciências Exatas. Nos dias atuais profissionais que atuam em áreas diversas, como Ciências Biológicas, Ciências da Saúde e até Ciências Humanas, acabam por utilizar essas ferramentas em seu cotidiano. Mesmo profissionais com formação em nível técnico eventualmente necessitam de Cálculo.

Apesar de ser necessário, o índice de reprovação nas disciplinas de Cálculo são tradicionalmente grandes nas instituições de ensino superior brasileiras. Segundo Silva e Souza (2014):

Sabe-se que o cálculo é de fundamental importância para os cursos de matemática, engenharia, física, ciências da computação, entre outros. Sendo assim, devemos levá-lo mais a sério, porque os alunos desses cursos entram nas universidades sem estar devidamente preparados, causando, então, um índice alarmante de desistências e reprovações. (Silva e Souza, 2014) 
Vários artigos relatam também a grande evasão dos cursos que incluem disciplinas de Cálculo no currículo. Em geral, tais números são associados aos altos índices de reprovação nessas disciplinas. A evasão é ocasionada por um conjuntos de fatores. Um deles é a dificuldade em operações básicas de matemática. Segundo Rezende (2003):

\begin{abstract}
....as dificuldades de aprendizagem relacionadas à operação de limite estão associadas muito mais às suas dificuldades em manipulações algébricas (fatoração de polinômios, relações trigonométricas, simplificações algébricas, "produtos notáveis" etc) do que à sua interpretação analítica (Rezende, 2003).
\end{abstract}

Ainda para Frescki e Pigatto (2009), é necessário um conhecimento prévio sólido de manipulações algébricas, operações e funções por parte dos alunos para que o ensino do cálculo tenha sucesso. Estudos recentes (Sadler e Sonert, 2018) têm demonstrado que, de fato, o domínio da matemática preparatória para Cálculo possui um impacto maior do que o ensino de Cálculo no Ensino Médio na performance dos estudantes em Cálculo no Ensino Superior. Isso mostra que o ensino de Cálculo como disciplina não pode substituir o ensino dos fundamentos matemáticos necessários. Por outro lado, como já dissemos, o Cálculo está cada vez mais presente em áreas que tradicionalmente não possuem essa disciplina nos respectivos currículos de ensino. Os profissionais que atuam nessas áreas acabam por utilizar as ferramentas do Cálculo, porém, sem ter um contato prévio com a disciplina.

Uma maneira de conciliar essas duas visões aparentemente antagônicas passa por uma maneira de tratar as dificuldades dos estudantes envolvendo operações matemáticas. Uma abordagem tradicional do ensino de Cálculo no Ensino Médio acaba passando obrigatoriamente pela etapa em que os estudantes precisam efetuar essas operações. Se os estudantes apresentarem dificuldades com as operações consequentemente deixarão de absorver o conteúdo de Cálculo.

Um modo de contornar essa dificuldade é através da utilização de recursos computacionais. softwares gratuitos oferecem a possibilidade de efetuar não somente cálculos numéricos, mas permitem também tratar dinamicamente o gráfico das funções e fazer cálculos algébricos de dificuldade moderada. Nesses softwares algumas operações básicas do Cálculo, como a derivação e a integração, podem ser efetuadas automaticamente, sem a necessidade de o estudante empreender esforço nessas operações.

A ideia deste trabalho é utilizar tais recursos para fornecer uma introdução ao Cálculo ao estudante do Ensino Médio. Através desta abordagem ele fica livre da necessidade de saber de antemão todo o ferramental algébrico e teórico do Cálculo e, ao mesmo tempo, é introduzido, de maneira intuitiva, aos diferentes conceitos. As operações matemáticas em si deverão ser tratadas separadamente. A utilização de softwares faz com que os estudantes possam construir seus próprios problemas e interagir com as soluções, deixando as operações matemáticas por conta do software. Os estudantes podem então continuar os estudos da matemática preparatória tendo como um dos objetivos a aprendizagem do Cálculo, independentemente da área que for seguir. Para aqueles que não forem seguir áreas em que o Cálculo faz parte do currículo, esta introdução pode ser útil caso exista a necessidade de utilizá-la posteriormente.

Este trabalho é dividido da seguinte forma. Na Seção 2 apresentamos um breve histórico sobre o ensino de Cálculo no Brasil. Na Seção 3 introduzimos os recursos computacionais como ferramentas no ensino de matemática. Na Seção 4 a definição de limite é explorada com a utilização do Geogebra. 
Na Seção 5 o wxMaxima é utilizado para explorações adicionais no cálculo de limites. Finalmente, na Seção 6 apresentamos nossa conclusões.

\section{O ensino de cálculo no Ensino Médio brasileiro}

O ensino no Brasil remonta o ano de 1549, sendo conduzido por pouco mais de duzentos anos pelos padres jesuítas. Segundo Miorim (1998), nessa época a educação era baseada na tradição clássico-humanista, sendo a matemática pouco estudada. A partir de 1722 foram criadas as aulas régias originadas da reforma pombalina. Essas aulas consistiam no ensino de disciplinas isoladas na forma de aulas avulsas, sem qualquer planejamento escolar. Disciplinas como Aritmética, Álgebra e Geometria eram introduzidas nas aulas régias. A matemática não se difundiu nessa época no Brasil, pois poucos alunos inscreviam-se nessas aulas. De um modo geral, as ciências exatas e naturais não eram vistas com bons olhos, e a formação do ensino secundário ainda era humanista.

O Primeiro Movimento Internacional para a modernização do ensino de matemática, teve início por volta de 1870. Criou-se a Comissão Internacional para o Ensino de Matemática, que propiciou a publicação de artigos voltados a tal área. Uma das maiores preocupações era com o ensino secundário, onde a matemática era ensinada em descompasso com o ensinado nas universidades. Entre as propostas para modificar o ensino secundário, estava a implementação do Cálculo ao currículo.

Em 1890, no Brasil República, Benjamin Constant, então ministro da Instrução, Correios e Telégrafos, deu origem a uma profunda reforma na educação. Houve a extinção da educação clássicohumanista, para a introdução de uma formação científica. A reforma seguiu a filosofia de Augusto Comte, o positivismo, na qual a matemática passava a ser de fundamental importância. O ensino secundário passou a ter sete anos, e o Cálculo Diferencial e Integral foi inserido no currículo, seguindo, em parte, a sugestão do Primeiro Movimento Internacional, pois era voltado apenas ao estudo de mecânica geral. A Reforma enfrentou diversas manifestações contrárias, e o ensino brasileiro passaria por várias reformas até 1930, sem que houvesse grandes mudanças. O ensino secundário continuava sendo considerado apenas como uma preparação para os cursos de medicina, direito e engenharia.

Em 1931, Francisco Campos dividiu o ensino secundário em dois ciclos. O primeiro era o ensino fundamental, com cinco anos de duração, e o segundo, complementar, com apenas dois anos. O ensino da matemática deixava de ter como objetivo apenas o desenvolvimento do raciocínio lógico. Ela começava a ser ensinada também com foco na aplicação em diversas áreas do conhecimento. Em relação à divisão do ensino secundário, na quarta e quinta série do ciclo fundamental, conceitos básicos de Cálculo Infinitesimal foram introduzidos. Na parte complementar o Cálculo fazia parte de dois programas, do pré-politécnico e do pré-médico, ficando de fora apenas do pré-jurídico. De acordo com Miorim (1998):

A proposta também trazia uma visão mais moderna dos conteúdos matemáticos, sugerindo a eliminação de "assuntos de interesse puramente formalístico", de "processos de cálculos desprovidos de interesse didático" e introduzindo o conceito de função e noções de cálculo infinitesimal"(Miorim, 1998).

O ensino do Cálculo na educação básica resistiu à reforma de Capanema em 1942, onde o ensino básico passou por uma reestruturação. O ensino secundário passou a ser dividido em dois ciclos 
paralelos, o Clássico e Científico. Nesta divisão estudantes que queriam seguir para a universidade cursavam o Clássico e os estudantes que queriam trabalhar após o ensino secundário, cursavam o Científico, o qual por sua vez era dividido em três cursos: Industrial, Comercial e Agrícola. O Cálculo ficou restrito ao Científico, sendo ensinado na terceira série derivadas, algumas aplicações e problemas de máximos e mínimos.

O Movimento Matemática Moderna na Europa e Estados Unidos ganhou força após a Segunda Guerra Mundial. Segundo Pinto (2005), esse movimento atingiu "não somente as finalidades do ensino, como também os conteúdos tradicionais da Matemática, atribuindo uma importância primordial à axiomatização, às estruturas algébricas, à lógica e aos conjuntos". Os seus efeitos chegaram ao Brasil no início da década de 60. A álgebra e o estudo da teoria dos conjuntos, com toda sua simbologia, foram priorizados. O Cálculo foi então excluído da educação básica, por não haver mais espaço no currículo, inchado pelo rigor e formalismo agora exigidos e ditos como modernos para o ensino da matemática. Assim, o que havia de mais moderno na matemática foi retirado da educação básica. Para Ávila (1991):

\begin{abstract}
Portanto, descartá-lo no ensino é grave, porque deixa de lado uma componente significativa e certamente a mais relevante da Matemática para a formação do aluno num contexto de ensino moderno e atual. Incorreram os reformistas naquele erro de recusar a pedra angular, aquela que seria a mais importante na construção do edifício... (Ávila, 1991)
\end{abstract}

Desde então, no Brasil, questões de Cálculo não são mais cobradas em vestibulares, e o mesmo foi abandonado como conteúdo a ser abordado no ensino médio e passou a ser exclusividade de cursos superiores.

\title{
3. Recursos Computacionais na Matemática
}

Os recursos computacionais estão cada vez mais presentes nas salas de aula, e ao ignorá-los corre-se o risco de "tornar a escola tão anacrônica em relação à vida exterior a seus muros a ponto de ter um efeito inócuo na formação dos alunos" (Giraldo, Caetano e Mattos, 2012). Contudo, a utilização indiscriminada dessas ferramentas pode impactar o ensino de forma negativa.

Para que essa metodologia seja bem-sucedida é preciso relacionar os conceitos e propriedades aprendidos normalmente de forma estanque em sala de aula com outras representações que podem ser fornecidas pela utilização dos recursos computacionais. Essa interação, segundo Giraldo, Caetano e Mattos (2012), reforça a ideia de que experiência concreta e o pensamento abstrato não precisam adquirir um caráter antagônico. As próprias limitações de cada recurso, como erros de arredondamento, podem ser exploradas de forma a aprofundar a discussão, sendo uma valiosa ferramenta pedagógica.

Neste trabalho iremos utilizar dois recursos computacionais (softwares) diferentes para a exploração dos conceitos de Cálculo. Os dois softwares utilizados serão o Geogebra e o wxMaxima, sendo o último uma interface gráfica para o Maxima. Cada um dos softwares utilizados servirá para um propósito específico dentro de cada tópico. Trataremos de ambos nas subseções seguintes. 


\subsection{Geogebra}

O Geogebra é um software livre de geometria dinâmica, álgebra e cálculo. Pode ser instalado também nos principais sistemas operacionais ou utilizado online através do site http://geogebra. org. Esse programa é de fácil exploração pelo usuário, pois possui uma interface mais amigável e intuitiva, sendo bastante difundido entre os professores de matemática. A Figura 1 mostra um exemplo de interface do programa.

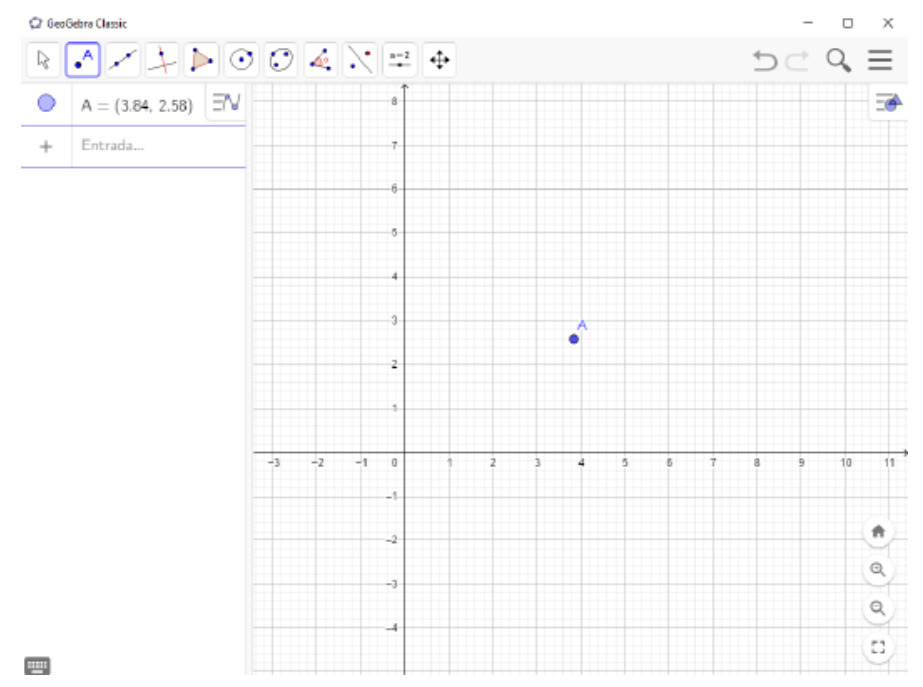

Figura 1: Tela do Geogebra. Os objetos e medidas podem ser construídos a partir de comandos ou acessados pelos botões na parte superior.

Em geral o Geogebra é utilizado para explorações envolvendo geometria plana (embora existam versões voltadas à geometria espacial). Ele inclui uma série de ferramentas para a exploração de funções reais, incluindo o cálculo de algumas derivadas e integrais. O resultado fornecido pelo Geogebra para essas duas operações é sempre numérico, o que não impede que possa ser utilizada para fornecer uma interpretação geométrica de tais operações.

\subsection{Maxima}

O Maxima (Vodopiec, 2018; Wikipedia, 2019) é um software classificado como Sistema de Computação Algébrica e Simbólica (SCAS). Isso significa que o programa é capaz de realizar cálculos numéricos e simbólicos, além de produzir gráficos em duas ou três dimensões. Existem softwares comerciais nessa mesma categoria que possuem recursos mais avançados. No entanto escolas públicas em geral não têm condições de arcar com o custo da aquisição e manutenção. O Maxima é um software livre, o que significa que pode ser instalado gratuitamente nos principais sistemas operacionais.

O Maxima é capaz de resolver equações e sistemas de forma simbólica, bem como operar com matrizes, construir gráficos a partir de funções, e calcular derivadas e primitivas de várias funções, 
o que permite utilizá-lo para efetuar operações algébricas necessárias ao Cálculo Diferencial e Integral, objetivo principal deste trabalho.

Trabalhar com o Maxima pode ser difícil no início, pois é necessário operar com uma série de comandos específicos, sendo, de certo modo, uma linguagem de programação. As versões mais novas do Maxima possuem uma interface gráfica mais amigável. Neste trabalho utilizaremos uma versão de interface gráfica chamada wxMaxima. A interface do wxMaxima está apresentada na Figura 2. O usuário inexperiente com os comandos pode se orientar pelo menu na parte superior deste programa, que oferece as ferramentas para fazer os cálculos desejados. Com um planejamento adequado da aula, o professor certamente terá sucesso em introduzir o uso do software.

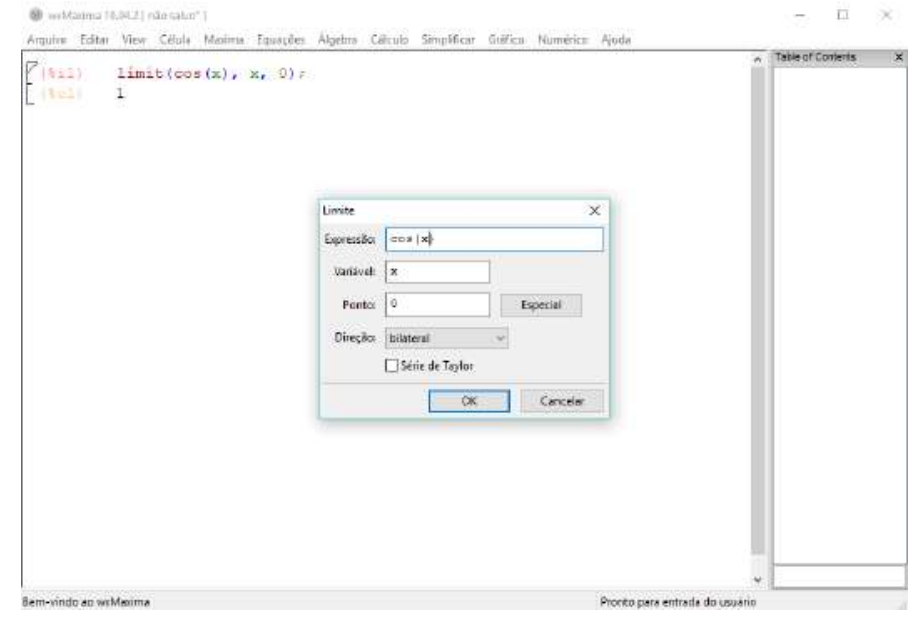

Figura 2: Tela do wxMaxima. O limite pode ser calculado utilizando um comando direto ou acessando o menu na barra da parte superior.

\section{Limites com o uso do Geogebra}

O conceito de limite de uma função real não é abordado no Ensino Médio pois o seu entendimento depende de um formalismo que é considerado avançado para o nível dos estudantes nessa etapa de formação. O conceito de ponto de acumulação de um conjunto, fundamental para a definição formal de limite, é bastante abstrato. Por exemplo, em um espaço topológico temos a seguinte definição:

Definição 1. Um ponto $P$ é um ponto de acumulação ou ponto limite de um conjunto $X$ se, para toda vizinhança de $P$, existe ao menos um ponto de $X$ diferente de $P$.

Apesar de o conceito de vizinhança ser abstrato, ele pode ser ilustrado para subconjuntos de $\mathbb{R}$ e $\mathbb{R}^{2}$ através do Geogebra, conforme ilustrado na Figura 3. Nessa atividade a vizinhança de um ponto é definida como o disco centrado nesse ponto. O conjunto $X$ é definido como toda a região na cor bege, incluindo o ponto isolado $D$. O controle deslizante na parte superior direita permite controlar o raio de todas as vizinhanças. Neste caso percebe-se que o ponto $A \in X$, por exemplo, é ponto de acumulação, pois toda vizinhança de $A$, excluindo-se o próprio $A$, possui pontos de $X$. O ponto $D \in X$, ao contrário, apesar de pertencer ao conjunto, não é ponto de 
acumulação, pois existe uma vizinhança tal que, excluindo-se $D$, não possui nenhum ponto de $X$. De maneira análoga temos pontos como o $B$, que não pertence a $X$ e não é ponto de acumulação. Por último, existem pontos como o ponto $C$, que não pertence ao conjunto (está na fronteira de um conjunto aberto) mas é ponto de acumulação, pois toda vizinhança de $C$ possui pontos de $X$. Essa construção é bastante simples e possui uma vantagem sobre uma figura estática, permitindo ao estudante interagir com a construção, mudando o raio dos discos através do controle deslizante. Dessa maneira o estudante pode perceber, por exemplo, que mesmo para pontos que não são de acumulação, existem vizinhanças que englobam pontos de $X$ sendo o raio $r$ suficientemente grande. Note que essa construção não substitui o formalismo envolvido, sendo meramente uma ilustração do conceito.

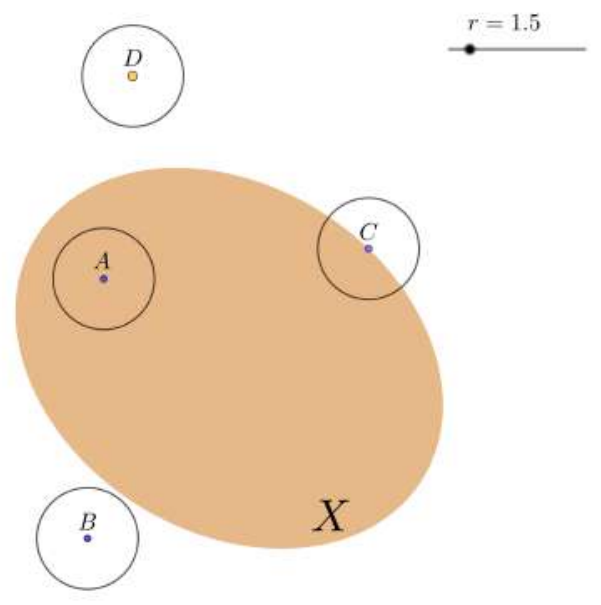

Figura 3: Figura do Geogebra contendo material sobre pontos de acumulação. O conjunto $X$ é definido pelos pontos de cor bege, incluindo o ponto $D$. O controle deslizante refere-se ao raio do círculo ao redor de cada ponto. Nesse caso, os pontos $A$ e $C$ são pontos de acumulação, mas não os pontos $B$ e $D$.

A definição formal de limite normalmente é um conceito de difícil assimilação pelos estudantes. É possível trabalhar com uma definição intuitiva como a fornecida por Stewart (2016):

Definição 2. Escrevemos

$$
\lim _{x \rightarrow a} f(x)=\ell
$$

e dizemos

"o limite de $f(x)$, quando $x$ tende a $a$, é igual a $\ell$ ",

se pudermos tomar os valores de $f(x)$ arbitrariamente próximos de $\ell$ (tão próximos de $L$ quanto quisermos), tomando $x$ suficientemente próximo de $a$ (por ambos os lados de $a$ ), mas não igual a $a$.

O problema dessa definição é que não ficam claros os conceitos arbitrariamente próximo e suficientemente próximo. Para um estudante interessado apenas nas ferramentas do Cálculo, tal noção 
é suficiente para que ele possa prosseguir no estudo das técnicas para obtenção de limites. Para o aluno de ensino médio interessado no conceito, essa definição pode causar alguma confusão. Tomemos a definição precisa, dada por Stewart (2016):

Definição 3. Seja $f$ uma função definida sobre algum intervalo aberto que contém o número $a$, exceto possivelmente o próprio $a$. Então dizemos que o limite de $f(x)$ quando $x$ tende a $a$, é $\ell$, e escrevemos

$$
\lim _{x \rightarrow a} f(x)=\ell,
$$

se, para todo número $\varepsilon>0$, houver um número $\delta>0$ tal que

$$
\text { se } 0<|x-a|<\delta \text { então }|f(x)-\ell|<\varepsilon .
$$

Apesar de mais abstratos, os conceitos arbitrariamente próximo e suficientemente próximo ficam claros com a introdução dos números $\varepsilon$ (um número positivo qualquer) e $\delta$ (um número adequado ao comportamento da função). O aluno de ensino médio em geral não possui o grau de capacitação necessário para demonstrar a existência de um determinado limite. A visualização deste conceito, porém, pode ser produtiva para este aluno.

Dado a um ponto de acumulação do domínio da função $f$, seja $L$ um número real. Vamos usar o software para testar se o limite de $f(x)$, quando $x$ tende a $a$ é $\ell$. Pela Definição 3 devemos ter $0<|x-a|<\delta$. Isto é equivalente a escrever

$$
a-\delta<x<a+\delta,
$$

ou seja, que $x$ pertence ao intervalo aberto $(a-\delta, a+\delta)$, com exceção do ponto $a$. De maneira análoga temos $|f(x)-\ell|<\varepsilon$, o que significa que $f(x)$ está no intervalo aberto $(\ell-\varepsilon, \ell+\varepsilon)$.

Vamos primeiramente trabalhar com uma função contínua, isto é tal que

$$
\lim _{x \rightarrow a} f(x)=f(a) .
$$

Escolhemos a função quadrática $f(x)=x^{2}$ por ser simples e ao mesmo tempo exibir a dependência de $\delta$ com o ponto $a$. A construção pode ser visualizada na Figura 4. Marcamos o ponto $A=(a, 0)$ no eixo $x$ e o ponto $L=(0, \ell)$. Os valores $\varepsilon$ e $\delta$ são controlados através de controles deslizantes. Dado um $\varepsilon>0$, construímos os pontos auxiliares $(0, \ell \pm \varepsilon)$ e traçamos duas retas perpendiculares ao eixo $y$ passando por tais pontos. Essas duas retas são fronteiras, portanto, do intervalo $(\ell-\varepsilon, \ell+\varepsilon)$. De maneira análoga construímos os pontos auxiliares $(a \pm \delta, 0)$, que são fronteira do intervalo aberto $(a-\delta, a+\delta)$. Sabendo que uma função contínua sempre possui um valor máximo e um valor mínimo em um intervalo fechado, o Geogebra permite avaliar o valor máximo e mínimo dessa função no intervalo $[a-\delta, a+\delta]$. Vamos denotar esses valores $\operatorname{Max}_{f}(a, \delta)$ e $\operatorname{Min}_{f}(a, \delta)$, respectivamente. Com estes valores podemos então avaliar a variação da função $f$ quando $x$ assume os valores permitidos dentro do intervalo $[a-\delta, a+\delta]$. Para mostrar visualmente a variação de $f$ no intervalo construímos um retângulo cujos vértices são os pontos cujas coordenadas são $\left(a \pm \delta, \operatorname{Max}_{f}(a, \delta)\right)$ e $\left(a \pm \delta, \operatorname{Min}_{f}(a, \delta)\right)$. A função $f$ terá limite $\ell$ para $x$ tendendo a $a$ se, para qualquer valor de $\varepsilon$ o retângulo gerado puder ser encaixado entre as duas retas escolhendo $\delta$ suficientemente pequeno. Nessa primeira construção os controles referentes aos valores $\varepsilon$ e $\delta$ são independentes para permitir ao estudante explorar esses intervalos, tentando encontrar manualmente um valor de $\delta$ para cada $\varepsilon$. 

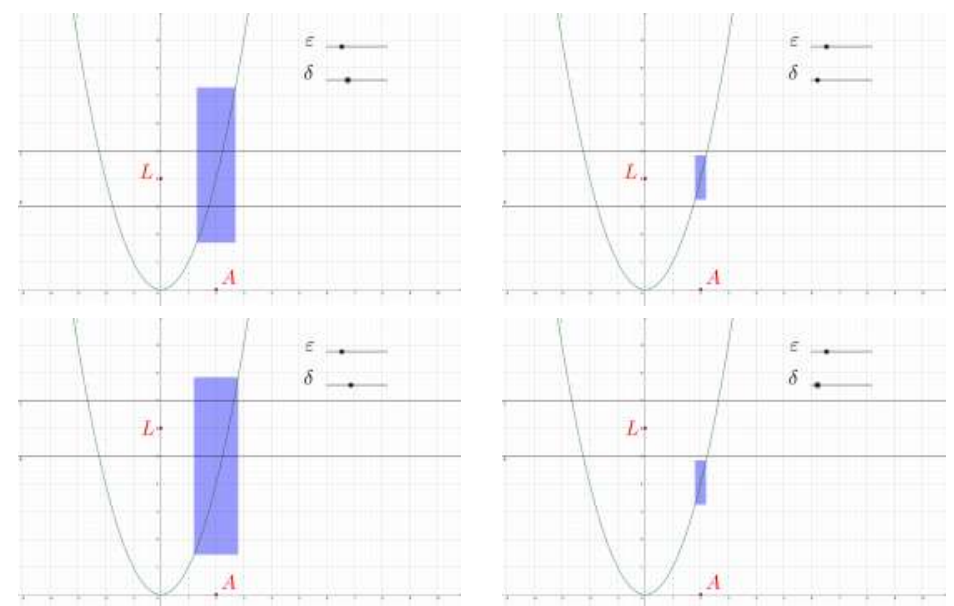

Figura 4: Visualização do conceito de limite utilizando o Geogebra para a função $f(x)=x^{2}$. O estudante pode explorar $\delta$ independentemente para verificar se a condição de limite é satisfeita para diferentes valores de $\varepsilon$. Temos que $\ell=4$ é limite de $f(x)=x^{2}$ quando $x$ tende a 2 . No primeiro caso (acima, à esquerda) o parâmetro $\delta$ não é pequeno o suficiente para que a variação de $f$ esteja totalmente contida no intervalo $(4-\epsilon, 4+\epsilon)$. Conforme, porém, o segundo caso (acima, à direita) o parâmetro $\delta$ pode sempre ser reduzido de forma a atender o requerimento. Temos que $\ell=6$ não é limite de $f(x)=x^{2}$ quando $x$ tende a 2 . No terceiro caso (abaixo, à esquerda) podemos tomar $\delta$ grande o bastante tal que alguns valores de $f$ estejam no intervalo $(6-\varepsilon, 6+\varepsilon)$. No entanto, para pequenos valores de $\varepsilon$, conforme o quarto caso (abaixo, à direita) a variação de $f$ nunca está totalmente dentro desse intervalo, não importando o valor de $\delta$.
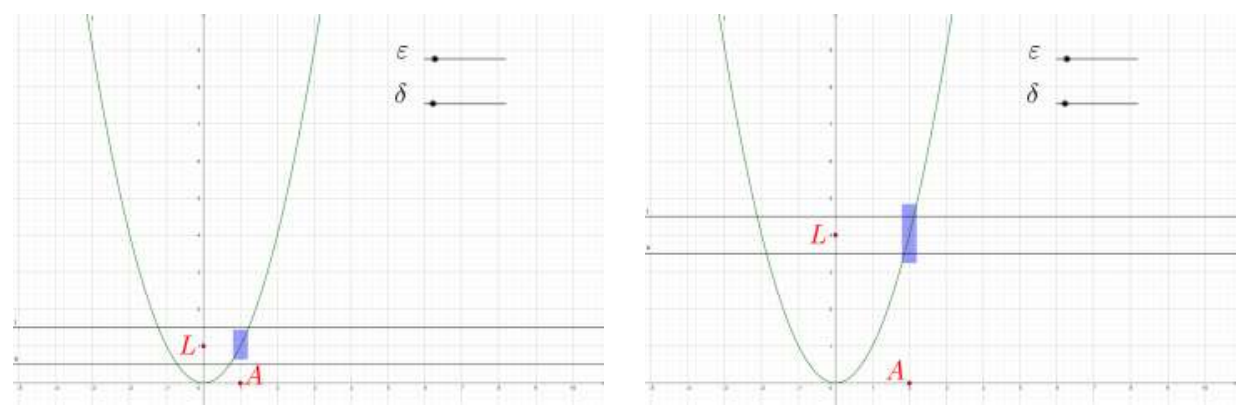

Figura 5: Dependência de $\delta$ para $f(x)=x^{2}$. No primeiro caso (à esquerda) temos que o limite para $x$ tendendo a $a=1$ é $\ell=1 \mathrm{e}$, para $\varepsilon=0,5$ é suficiente tomar $\delta=0,2$. No segundo caso (à direita) temos que o limite para $x$ tendendo a $a=2$ é $\ell=4$, mas para $\varepsilon=0,5$ o valor $\delta=0,2$ não é suficiente para termos $|f(x)-\ell|<\varepsilon$.

Para demonstrar que $\lim _{x \rightarrow a} x^{2}=a^{2}$, dado $\varepsilon>0$, basta tomar $\delta$ conforme a expressão

$$
\delta=\min \left\{1, \frac{\epsilon}{1+2|a|}\right\}
$$


Se $|x-a|<\delta$ vale a seguinte identidade:

$$
|x+a|=|x-a+2 a| \leq|x-a|+|2 a|<1+2|a| .
$$

Portanto temos, finalmente,

$$
\left|x^{2}-a^{2}\right|=|x-a||x+a|<\delta(1+2|a|) \leq \epsilon,
$$

o que finaliza a demonstração.

A demonstração pode ser difícil para um aluno do Ensino Médio seguir, porém ela carrega alguns aspectos interessantes. O primeiro aspecto é o fato da dependência de $\delta$ do ponto $a$ ficar explícita através da Equação 1. Essa dependência pode ser visualizada através do Geogebra fixando $\varepsilon$ e $\delta$. Para diferentes valores $a$, o valor $\delta$ utilizado pode não ser suficientemente pequeno para o retângulo ficar contido entre as linhas, como mostra a Figura 5. Outro aspecto interessante é que o valor $\delta$ necessário é fornecido na demonstração como função de $\varepsilon$ e $a$. Esse valor pode ser implementado no Geogebra de forma a termos um valor $\delta$ fornecido automaticamente para $\varepsilon$ e $a$ dados. O resultado pode ser visto na Figura 6.

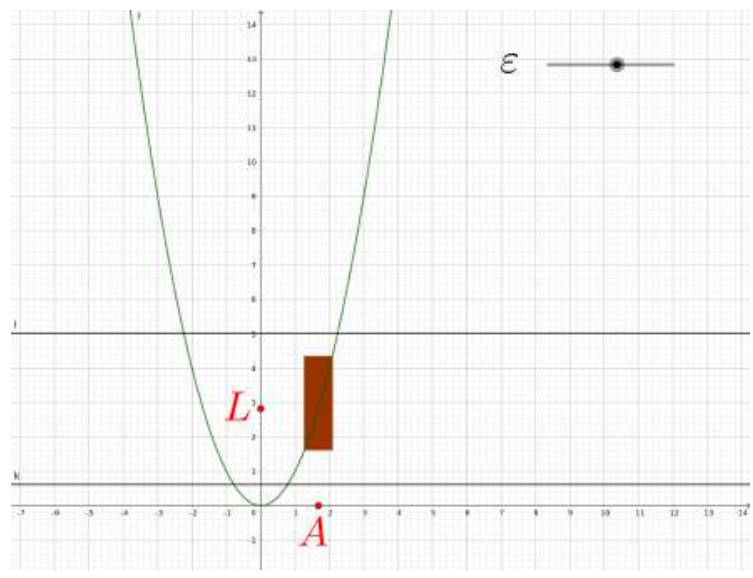

Figura 6: Visualização da demonstração de que $\lim _{x \rightarrow a} x^{2}=a^{2}$. O valor de $\delta$ dado através da Equação 1 pode ser reduzido para facilitar a visualização do conceito, que envolve uma desigualdade restrita. Nesse caso escolhemos trabalhar com $80 \%$ de tal valor, mas qualquer redução é válida, desde que o número permaneça positivo.

É preciso tomar alguns cuidados nessa construção. Entre os principais está o fato de que os intervalos envolvidos na definição de limite são abertos, e a função não precisa estar necessariamente definida em $a$. Por isso é importante utilizar tal construção com vários tipos de função, com características distintas.

Vamos analisar a função $g: \mathbb{R} \rightarrow \mathbb{R}$ dada por $g(x)=x^{2}$ para $x \neq 2$ e $g(2)=6$. Note que essa função corresponde à mesma função $f$ estudada anteriormente, salvo em $x=2$, em que $g$ corresponde a um valor diferente. A função $g$ é implementada facilmente no Geogebra utilizando o comando Se. Conforme pode ser visto na Figura $7, \lim _{x \rightarrow 2} g(x)=4$, mesmo que $g(2)$ seja 6 . Este exemplo é 
importante pois mostra que o limite de uma função em um determinado ponto é independente do valor da função nesse ponto. De fato, mesmo que a função não estivesse definida nele definida o limite continuaria a existir.

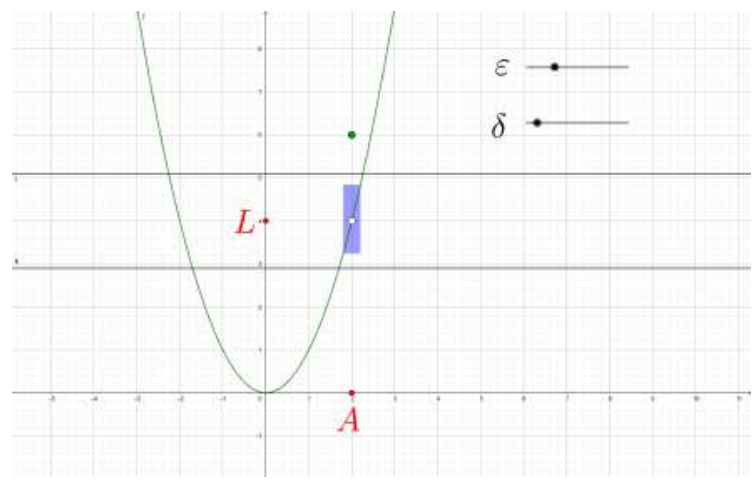

Figura 7: Caso ilustrando o caso de uma função descontínua em que o limite existe. Neste caso tem-se $g(2)=6$ e $\lim _{x \rightarrow 2} g(x)=4$.

A seguir, vamos considerar a função $h: \mathbb{R} \rightarrow \mathbb{R}$ definida por

$$
h(x)=\left\{\begin{array}{cc}
x^{2} & \text { se } x<0 \\
x^{2}+1 & \text { se } x \geq 0
\end{array}\right.
$$

Esta função também pode ser implementada no Geogebra através do comando Se. Temos que $h(0)=1$ por definição. Vamos verificar o que ocorre no limite de $x$ tendendo a 0. Fazendo $a=0$ na construção do Geogebra verifica-se que a altura do retângulo de variação de $h$ no intervalo $(-\delta, \delta)$ sempre será no mínimo 1 . Isso ocorre porque nesse intervalo, não importando o valor $\delta>0$, existem sempre valores próximos de zero e maiores do que 1, de forma que $\operatorname{Max}_{h}(0, \delta)=1$ e $\operatorname{Min}_{h}(0, \delta)=0$. Considerando $\varepsilon=1 / 2$, por exemplo, a variação de $h$ estará sempre fora do intervalo $(\ell-\varepsilon, \ell+\varepsilon)$, não importando o valor de $\ell$. Isso nos leva a concluir que o limite de $h(x)$ quando $x$ tende a 0 não existe.

A exploração de limites de diferentes funções também é possível com essa construção no Geogebra. Fica claro, portanto, a interatividade e flexibilidade fornecidas pela ferramenta computacional em detrimento de exemplos estáticos. É necessário deixar claro ao aluno que a exploração dos casos através da ferramenta computacional é um auxílio à visualização destes conceitos, mas não substitui uma demonstração formal.

\section{Limites com o uso do wxMaxima}

O wxMaxima pode ser utilizado de forma complementar para efetivamente calcular limites. A ideia é utilizá-lo para fornecer ao estudante um método de cálculo que permita a reflexão dos resultados que são mostrados na tela. O comando para calcular limites no xwMaxima é o limit, utilizando como primeiro argumento a função, o segundo a variável e terceiro o valor em que se deseja calcular o limite. Por exemplo, ao calcular o limite da função $f(x)=x^{2}$ acima quando $x$ tende a 0 , temos o resultado abaixo: 

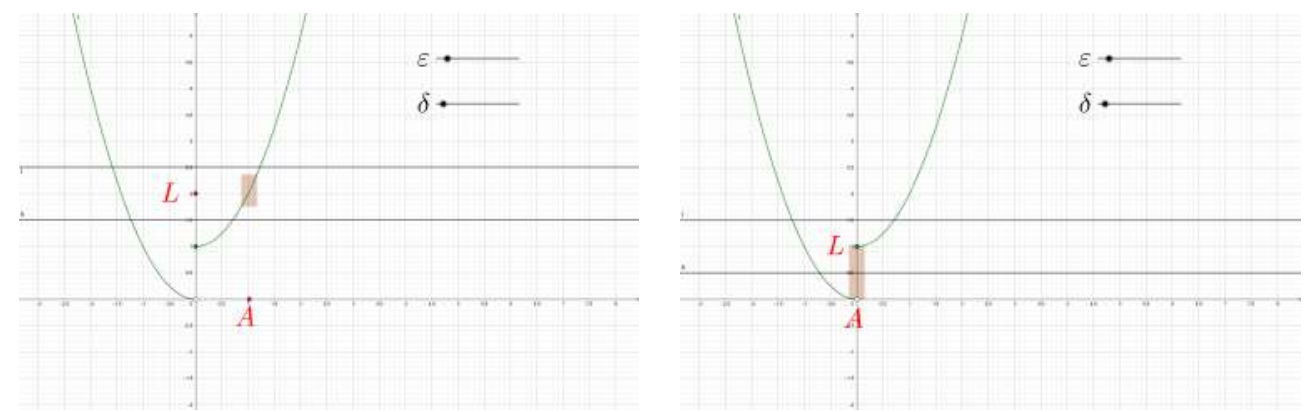

Figura 8: Exploração de uma função descontínua que não possui limite somente no ponto de descontinuidade. Na figura à esquerda, ilustra-se um ponto em que o limite existe e corresponde a $h(x)$. Na figura à direita, ao se utilizar o ponto de descontinuidade $(x=0)$, fica claro que apesar de a função estar definida no ponto $(h(0)=1)$, o limite da função não pode corresponder a esse valor. Usando outros candidatos ao valor do limite pode-se verificar que o mesmo não existe.

$\left(\%\right.$ i1) $\quad f(x):=x^{\wedge} 2$;

$\operatorname{limit}(f(x), x, 0)$;

$(\% \mathrm{o} 1) \mathrm{f}(x):=x^{2}$

(\%o2) 0 A utilidade do wxMaxima, porém, vai além de simplesmente calcular expressões numéricas. Como ferramenta de cálculo algébrico e simbólico, podemos comparar as expressões escritas em termos de variáveis. Por exemplo, no caso anterior, suponha que desejamos saber se $\lim _{x \rightarrow a} f(x)=a^{2}$. Podemos comparar as expressões utilizando os comandos is e equal conforme abaixo:

$\left(\%\right.$ i2) is (equal ( $\left.\left.\operatorname{limit}(f(x), x, a), a^{\wedge} 2\right)\right)$;

(\%o2) true Caso substituíssemos $a^{2}$ por -1 teríamos

$(\%$ i3) is (equal $(\operatorname{limit}(f(x), x, a),-1))$;

(\%o3) false De fato, o limite de $f$ nunca poderá ser um número negativo. Contudo, se tentarmos a expressão $a^{3}$ obtemos o seguinte resultado:

$\left(\%\right.$ i4) is (equal $\left.\left(\operatorname{limit}(f(x), x, a), a^{\wedge} 3\right)\right)$;

(\%o4) unknown $\mathrm{O}$ resultado unknown (desconhecido) é interessante, pois pode levar os estudantes à reflexão de como o software faz as operações de cálculo e comparação. O software não fornece uma negativa neste caso, pois existe pelo menos um caso em que vale a igualdade $(a=0)$. Vamos utilizar agora a função $g$ definida na seção anterior. A função pode ser definida através do seguinte comando:

(\%i5) $g(x):=$ if $x=2$ then 6 else $x^{\wedge} 2$;

$(\% \mathrm{o} 5) \mathrm{g}(x):=$ if $x=2$ then 6 else $x^{2}$ Este comando do wxMaxima pode ser lido da seguinte 
maneira: Se $x=2$, então $g(x)=6$, caso contrário, $g(x)=x^{2}$. Utilizando o comando limit é possível obter o limite dessa função e o valor da função no ponto $x=2$. O limite é diferente do valor do ponto, porém ele existe.

(\%i6) $g(2)$;

$(\%$ o6) 6

(\%i7) $\operatorname{limit}(\mathrm{g}(\mathrm{x}), \mathrm{x}, 2)$;

(\%o7) 4 A função $h$ também pode ser representada no wxMaxima. Para isso, faremos uso da função degrau, definida por

$$
s(x)= \begin{cases}1 & \text { se } x>0 \\ 0 & \text { se } x<0 .\end{cases}
$$

No wxMaxima essa função é representada por

$(\%$ i8) $\quad \mathrm{s}(\mathrm{x}):=(1 / 2) *(\mathrm{x} /(\operatorname{abs}(\mathrm{x}))+1)$;

$\left(\%\right.$ o8) $\mathrm{s}(x):=\frac{1}{2}\left(\frac{x}{|x|}+1\right)$ A função $h(x)$ pode ser então introduzida através do seguinte comando:

(\%i9) $\quad h(x):=$ if $x=0$ then 1 else $s(-x) * x \backslash \sim 2+s(x) *(x \backslash \sim 2+1)$;

$\left(\%\right.$ o9) $\mathrm{h}(x):=$ if $x=0$ then 1 else $\mathrm{s}(-x) x^{2}+\mathrm{s}(x)\left(x^{2}+1\right)$ Ao executar o comando para que o $w x-$ Maxima encontre o limite de $h$ quando $x$ tende a 2 , recebe-se o resultado correto.

$(\%$ i10) $\operatorname{limit}(\mathrm{h}(\mathrm{x}), \mathrm{x}, 2)$;

$(\% \mathrm{o} 10) 5$

Note, no entanto, que ao tentarmos obter o limite de $h$ para $x$ tendendo a zero obtemos o seguinte resultado.

$(\%$ i11) $\operatorname{limit}(\mathrm{h}(\mathrm{x}), \mathrm{x}, 0)$;

(\%o11) und Apesar de não existir o limite, pode-se verificar que $h(0)$ está definido na expressão do wxMaxima.

$(\%$ i12) $\mathrm{h}(0)$;

(\%o12) 1 A expressão und é uma abreviação para undefined (indefinido). Para entender o significado dessa expressão é necessário entender o significado de limite lateral.

Uma função $f$ possui limite lateral à direita

$$
\lim _{x \rightarrow a^{+}} f(x)
$$

se existe o limite da função considerando apenas valores à direita de $a$, ou seja, $x>a$. Analoga- 
mente, uma função $f$ possui limite lateral à esquerda

$$
\lim _{x \rightarrow a^{-}} f(x)
$$

se existe o limite da função considerando apenas valores à esquerda de $a$, ou seja, $x<a$. Para que o limite da função exista, os limites à esquerda e à direita devem existir e ser iguais.

No caso da função $h$ os limites laterais em $x=0$ existem, conforme é possível verificar pelo software. Porém, não são iguais.

$(\%$ i13) $\operatorname{limit}(\mathrm{h}(\mathrm{x}), \mathrm{x}, 0, \mathrm{plus})$;

(\%i14) $\operatorname{limit}(\mathrm{h}(\mathrm{x}), \mathrm{x}, 0$, minus);

(\%o13) 1

(\%o14) 0 A utilização do wxMaxima pode abranger as operações de limites e o cálculo de limites de grande dificuldade algébrica. Pode, também, ser utilizado para obter derivadas a partir da definição em uma aula introdutória. Contudo, a utilização os resultados obtidos devem ser sempre investigados quanto à sua validade, evitando aceitá-los sem uma verificação.

\section{Conclusões}

Neste artigo foi apresentada uma metodologia baseada na utilização de recursos computacionais para a introdução de limites no Ensino Médio. É importante reforçar que essa metodologia não substitui o ensino de Cálculo nos cursos superiores voltados às áreas de exatas. É possível, porém, ilustrar os conceitos através da sua realização utilizando alguns exemplos pré-escolhidos. Esses exemplos permitem que os estudantes absorvam estes conceitos e os confrontem ao aprender o formalismo matemático envolvido. Para os estudantes que não seguirão a área de exatas, a simples exposição aos conceitos pode ser interessante nas suas respectivas áreas de trabalho.

\section{Agradecimentos}

O presente trabalho foi realizado com apoio da Coordenação de Aperfeiçoamento de Pessoal de Nível Superior - Brasil (Capes) - Código de Financiamento 001. Agradecemos ao Programa de Mestrado Profissional em Matemática em Rede Nacional - Profmat.

\section{Referências}

[1] Ávila, G. O ensino de cálculo no segundo grau. Revista do Professor de Matemática, n. 18, p. $1-9,1991$.

[2] Frescki, F. B. e Pigatto, P. Dificuldades na aprendizagem de cálculo diferencial e integral na educação tecnológica: proposta de um curso de nivelamento. I Simpósio Nacional de Ensino de Ciência e Tecnologia, p. 910-917, 2009.

[3] Giraldo, V., Caetano, P. e Mattos, F. Recursos Computacionais no Ensino de Matemática. 1. ed. Rio de Janeiro: SBM, 2012.

[4] MAXIMA. In: WIKIPÉDIA, a enciclopédia livre. Flórida: Wikimedia Foundation, 2019. Disponível em: https://pt.wikipedia.org/w/index.php?title=Maxima\&oldid=55039853. Acesso em: 5 mai. 2019. 
[5] Vodopivec, A. Maxima, a Computer Algebra System. Versão 18.02.0, 2018. Disponível em http://maxima.sourceforge.net/.

[6] Miorim, M. A. Introdução a história da educação matemática. São Paulo: Atual, 1998.

[7] Pinto, N. B. Marcas históricas da matemática moderna no Brasil. Revista Diálogo Educacional, v. 5, n. 16, p. 25-38, 2005.

[8] Rezende, W. M. O Ensino de Cálculo: Dificuldades de natureza epistemológica. Tese (Doutorado) - Universidade de São Paulo, São Paulo, 2003.

[9] Sadler P. e Sonnert G. The Path to College Calculus: The Impact of High School Mathematics Coursework. Journal for Research in Mathematics Education, v. 49, n. 3 , p. 292-329, 2018.

[10] Silva, C. R. e Souza, K. R. Q. Cálculo: Uma proposta possível para o ensino médio. Revista Panorâmica On-Line, v. 17, p. 81-89, 2014.

[11] Stewart, J. Cálculo. $3^{a}$ ed. São Paulo: Cengage Learning, 2016, v. 1.

\section{A. Construção Geogebra}

Para construção da rotinas utilizadas apresentamos os passos detalhados utilizados no Geogebra. Primeiramente vamos construir a rotina de exploração da função $f$ dada por $f(x)=x^{2}$.

i) Na caixa de entrada, digite a expressão da função:

$$
f(x)=x \wedge 2
$$

ii) Defina os controles deslizantes $\varepsilon$ e $\delta$, tomando o cuidado de escolher o intervalo de definição de ambas positivo.

iii) Defina os pontos $A$ restrita eixo $x$ e $L$ restrito ao eixo $y$. A abcissa do ponto $A$ fornecerá o valor $a$, onde será avaliado o limite. A ordenada de $L$ corresponderá ao limite e deve ser encontrado pelo estudante.

iv) Defina, através da caixa de entrada, os pontos

$-A_{1}=(x(A)-\delta, 0), A_{2}=(x(A)+\delta, 0)$,

$-L_{1}=(0, y(L)-\varepsilon), L_{2}=(0, y(L)+\varepsilon)$,

- $P\left(x\left(A_{2}\right), y\left(\operatorname{Maximo}\left(f, x\left(A_{1}\right), x\left(A_{2}\right)\right)\right)\right)$ e $R\left(x\left(A_{1}\right), y\left(\operatorname{Minimo}\left(f, x\left(A_{1}\right), x\left(A_{2}\right)\right)\right)\right)$.

v) Defina, através da caixa de entrada, as retas

$-g=\operatorname{Perpendicular}\left(A_{1}\right.$, EixoX), $h=\operatorname{Perpendicular}\left(A_{2}\right.$, EixoX),

$-i=\operatorname{Perpendicular}\left(L_{1}\right.$, EixoY $), j=\operatorname{Perpendicular}\left(L_{2}\right.$, EixoY $)$,

$-k=\operatorname{Perpendicular}(P$, EixoY) e $l=\operatorname{Perpendicular}(R, E i x o Y)$.

vi) Defina, através da caixa de entrada, os pontos $Q=\operatorname{Intersecao}(g, k)$ e $S=\operatorname{Intersecao}(l, h)$ e defina o retângulo $P Q R S$. 
Essa construção permite ao estudante selecionar um ponto $a$ (manipulando $A$ ) e escolher um candidato a limite da função (manipulando $L$ ). Através da manipulação das caixas deslizantes, então o estudante pode verificar se o valor escolhido corresponde realmente ao limite, modificando os valores de $\varepsilon$ e verificando se, a partir de certo valor de $\delta$ suficientemente pequeno, a variação de $f$ restringe-se ao intervalo $(a-\varepsilon, a+\varepsilon)$.

Note que a função pode ser modificada sem prejuízo da construção. Por exemplo, para construir as funções $g$ e $h$ pode-se utilizar os seguintes comandos:

- $g(x)=S e\left(x=2,6, x^{2}\right)$

- $h(x)=S e(x<0, x \wedge 2, x \wedge 2+1)$

O comando Se do Geogebra possui a seguinte estrutura. Na primeira entrada coloca-se a condição, na segunda coloca-se o valor a ser retornado se a condição é satisfeita, e na terceira, o que retornar se a condição não é satisfeita. Para incluir mais de uma condição pode-se colocar outros comandos Se nas entradas.

Em exemplos em vez de o estudante poder obter o valor de $\delta$ automaticamente. Nesse caso, basta retirar o controle deslizante referente a $\delta$ e substituir o valor pela expressão correspondente. Por exemplo, no caso em que $f(x)=x^{2}$ o valor de $\delta$ deve ser menor do que $\min \left\{1, \frac{\epsilon}{1+2|a|}\right\}$. No Geogebra pode-se implementar

- $\delta=0.8 * \operatorname{Min}(1, \varepsilon /(1+2 * x(A))$

Sendo o valor 0,8 apenas para facilitar a visualização da desigualdade estrita.

É interessante observar também que é possível renomear os pontos e as retas de acordo com a necessidade do professor, clicando com o botão direito do mouse e entrando em propriedade. Além disso, para que a imagem fique mais limpa, basta desabilitar os pontos ou retas, basta clicar em cima dos mesmo na parte esquerda da interface do Geogebra.

Helena Corrêa Ribeiro

Universidade Tecnológica Federal do Paraná $<$ helenaribeiro09@hotmail.com>

André Fabiano Steklain Lisbôa Universidade Tecnológica Federal do Paraná <steklain@utfpr.edu.br> 